\title{
Alternative proofs for summation formulas of some trigonometric series
}

\author{
Feng $\mathbf{Q i}^{1,2,3,{ }^{*}}$ and Bai-Ni Guo ${ }^{4}$ \\ ${ }^{1}$ Institute of Mathematics, Henan Polytechnic University, Jiaozuo City, Henan Province, 454010, China \\ ${ }^{2}$ College of Mathematics, Inner Mongolia University for Nationalities, Tongliao City, Inner Mongolia Autonomous Region, China \\ ${ }^{3}$ Department of Mathematics, College of Science, Tianjin Polytechnic University, Tianjin City, 300387, China \\ ${ }^{4}$ School of Mathematics and Informatics, Henan Polytechnic University, Jiaozuo City, Henan Province, 454010, China \\ *Corresponding author E-mail: qifeng618@gmail.com
}

\begin{abstract}
In the paper, the authors supply alternative proofs for some summation formulas of trigonometric series.
\end{abstract}

Keywords: summation formula; trigonometric series; alternative proof.

\section{Introduction}

In 2016, after establishing

$$
\begin{aligned}
\sum_{n=1}^{\infty} 4^{n} \sin ^{4} \frac{x}{2^{n}} & =x^{2}-\sin ^{2} x \\
\sum_{n=1}^{\infty} \frac{\sin ^{4}\left(2^{n} x\right)}{4^{n}} & =\frac{\sin ^{2}(2 x)}{4} \\
\sum_{n=1}^{\infty}(-1)^{n} \frac{\cos ^{4}\left(2^{n} x\right)}{4^{n}} & =-\frac{3}{40}-\frac{\cos (4 x)}{8} \\
\sum_{n=1}^{\infty} 3^{n} \sin ^{3} \frac{x}{3^{n}} & =\frac{3}{4}(x-\sin x) \\
\sum_{n=1}^{\infty} \frac{\sin ^{3}\left(3^{n} x\right)}{3^{n}} & =\frac{\sin (3 x)}{4} \\
\sum_{n=1}^{\infty}(-1)^{n} \frac{\cos ^{3}\left(3^{n} x\right)}{3^{n}} & =-\frac{\cos (3 x)}{4}
\end{aligned}
$$

by means of telescoping method and after listing some formulas from [2, p. 39], Chu [1] posed that there may exist summation formulas for the trigonometric series

$$
U(\alpha, \beta ; x)=\sum_{n=1}^{\infty} \alpha^{n} \sin \left(\beta^{n} x\right)
$$

and

$V(\alpha, \beta ; x)=\sum_{n=1}^{\infty} \alpha^{n} \cos \left(\beta^{n} x\right)$,

where $\alpha, \beta \notin\{0, \pm 1\}$ and $x \neq 0$ are real constants.

It is obvious that

$V(\alpha, \beta ; x) \pm i U(\alpha, \beta ; x)=\sum_{n=1}^{\infty} \alpha^{n} e^{ \pm i \beta^{n} x}$ where $i$ denotes the imaginary unit in the complex numbers. For $\alpha, \beta \notin\{0, \pm 1\}, \gamma \in \mathbb{N}, x \neq 0$, and $m \in \mathbb{N} \cup\{\infty\}$, let

$U(\alpha, \beta, \gamma ; x ; m)=\sum_{n=1}^{m} \alpha^{n} \sin ^{\gamma}\left(\beta^{n} x\right)$

and

$V(\alpha, \beta, \gamma ; x ; m)=\sum_{n=1}^{m} \alpha^{n} \cos ^{\gamma}\left(\beta^{n} x\right)$.

It is clear that

1. $U(\alpha, \beta, 1 ; x ; \infty)=U(\alpha, \beta ; x)$ and $V(\alpha, \beta, 1 ; x ; \infty)=V(\alpha, \beta ; x)$;

2. when $0<|\alpha|<1$, the infinite trigonometric series $U(\alpha, \beta, \gamma ; x ; \infty)$ and $V(\alpha, \beta, \gamma ; x ; \infty)$ are absolutely convergent;

3. when $0<|\beta|<1$ and $0<\left|\alpha \beta^{\gamma}\right|<1$, the infinite trigonometric series $U(\alpha, \beta, \gamma ; x ; \infty)$ is absolutely convergent.

In this paper, we supply alternative proofs for summation formulas (1.1) to (1.6) by means of discussing the trigonometric series $U(\alpha, \beta, \gamma ; x ; \infty)$ and $V(\alpha, \beta, \gamma ; x ; \infty)$.

\section{Alternative proofs of the formulas to $(1.6)$}

We now start out to prove those formulas (1.1) to (1.6) alternatively. In [3, Corollary 2.1], Guo and Qi obtained

$\cos ^{\ell} x=\frac{1}{2^{\ell}} \sum_{q=0}^{\ell}\left(\begin{array}{l}\ell \\ q\end{array}\right) \cos [(2 q-\ell) x]$

and

$\sin ^{\ell} x=\frac{(-1)^{\ell}}{2^{\ell}} \sum_{q=0}^{\ell}(-1)^{q}\left(\begin{array}{l}\ell \\ q\end{array}\right) \cos \left[(2 q-\ell) x-\frac{\ell}{2} \pi\right]$ 
for $\ell \in \mathbb{N}$. Accordingly, when $\gamma \in \mathbb{N}$, we have

$U(\alpha, \beta, \gamma ; x ; \infty)$

$$
\begin{aligned}
& =\frac{(-1)^{\gamma} \sum_{q=0}^{\gamma}(-1)^{q}\left(\begin{array}{l}
\gamma \\
q
\end{array}\right) \sum_{n=1}^{\infty} \alpha^{n} \cos \left[(2 q-\gamma) \beta^{n} x-\frac{\gamma}{2} \pi\right]}{} \\
& =\left\{\begin{array}{l}
\frac{(-1)^{\gamma}}{2^{\gamma}} \sum_{q=0}^{\gamma}(-1)^{q}\left(\begin{array}{l}
\gamma \\
q
\end{array}\right) \sum_{n=1}^{\infty} \alpha^{n} \sin \left[(2 q-\gamma) \beta^{n} x\right], \\
\gamma=4 k+1 ; \\
\frac{(-1)^{\gamma+1}}{2^{\gamma}} \sum_{q=0}^{\gamma}(-1)^{q}\left(\begin{array}{l}
\gamma \\
q
\end{array}\right) \sum_{n=1}^{\infty} \alpha^{n} \cos \left[(2 q-\gamma) \beta^{n} x\right], \\
\frac{(-1)^{\gamma+1}}{2^{\gamma}} \sum_{q=0}^{\gamma}(-1)^{q}\left(\begin{array}{l}
\gamma \\
q
\end{array}\right) \sum_{n=1}^{\infty} \alpha^{n} \sin \left[(2 q-\gamma) \beta^{n} x\right], \\
\gamma=4 k+3 ; \\
\frac{(-1)^{\gamma}}{2^{\gamma}} \sum_{q=0}^{\gamma}(-1)^{q}\left(\begin{array}{l}
\gamma \\
q
\end{array}\right) \sum_{n=1}^{\infty} \alpha^{n} \cos \left[(2 q-\gamma) \beta^{n} x\right], \\
\gamma=4 k+4
\end{array}\right.
\end{aligned}
$$

and

$$
V(\alpha, \beta, \gamma ; x ; \infty)=\frac{1}{2 \gamma} \sum_{q=0}^{\gamma}\left(\begin{array}{l}
\gamma \\
q
\end{array}\right) \sum_{n=1}^{\infty} \alpha^{n} \cos \left[(2 q-\gamma) \beta^{n} x\right]
$$

for $k \in\{0\} \cup \mathbb{N}$. Consequently, taking $\alpha=\frac{1}{4}, \beta=2$, and $\gamma=4$ in the above expression for $U(\alpha, \beta, \gamma ; x ; \infty)$ leads to

$U\left(\frac{1}{4}, 2,4 ; x ; \infty\right)=\frac{1}{16} \sum_{q=0}^{4}(-1)^{q}\left(\begin{array}{l}4 \\ q\end{array}\right) \sum_{n=1}^{\infty} \frac{1}{4^{n}} \cos \left[(2 q-4) 2^{n} x\right]$

$=\frac{1}{16}\left[2 \sum_{n=1}^{\infty} \frac{1}{4^{n}} \cos \left(2^{n+2} x\right)-8 \sum_{n=1}^{\infty} \frac{1}{4^{n}} \cos \left(2^{n+1} x\right)+\left(\begin{array}{l}4 \\ 2\end{array}\right) \sum_{n=1}^{\infty} \frac{1}{4^{n}}\right]$

$=\frac{1}{16}\left[2 \sum_{n=1}^{\infty} \frac{1}{4^{n}} \cos \left(2^{n+2} x\right)-8 \sum_{n=0}^{\infty} \frac{1}{4^{n+1}} \cos \left(2^{n+2} x\right)+2\right]$

$=\frac{1}{16}[-2 \cos (4 x)+2]=\frac{1}{8}[1-\cos (4 x)]=\frac{\sin ^{2}(2 x)}{4}$.

The formula (1.2) is thus recovered.

Similarly, we have

$$
\begin{aligned}
& V\left(-\frac{1}{4}, 2,4 ; x ; \infty\right)=\frac{1}{16} \sum_{q=0}^{4}\left(\begin{array}{l}
4 \\
q
\end{array}\right) \sum_{n=1}^{\infty}(-1)^{n} \frac{1}{4^{n}} \cos \left[(2 q-4) 2^{n} x\right] \\
& =\frac{1}{16}\left[2 \sum_{n=1}^{\infty} \frac{(-1)^{n}}{4^{n}} \cos \left(2^{n+2} x\right)+8 \sum_{n=1}^{\infty} \frac{(-1)^{n}}{4^{n}} \cos \left(2^{n+1} x\right)\right. \\
& \left.\quad+\left(\begin{array}{l}
4 \\
2
\end{array}\right) \sum_{n=1}^{\infty} \frac{(-1)^{n}}{4^{n}}\right] \\
& =\frac{1}{16}\left[2 \sum_{n=1}^{\infty} \frac{(-1)^{n}}{4^{n}} \cos \left(2^{n+2} x\right)+8 \sum_{n=0}^{\infty} \frac{(-1)^{n+1}}{4^{n+1}} \cos \left(2^{n+2} x\right)-\frac{6}{5}\right] \\
& =\frac{1}{16}\left[-2 \cos (4 x)-\frac{6}{5}\right]=-\frac{3}{40}-\frac{\cos (4 x)}{8} .
\end{aligned}
$$

The formula (1.3) is thus proved again.

Furthermore, we have

$$
\begin{aligned}
& U\left(\frac{1}{3}, 3,3 ; x ; \infty\right)=\frac{1}{2^{3}} \sum_{q=0}^{3}(-1)^{q}\left(\begin{array}{l}
3 \\
q
\end{array}\right) \sum_{n=1}^{\infty} \frac{1}{3^{n}} \sin \left[(2 q-3) 3^{n} x\right] \\
& =\frac{1}{4}\left[-\sum_{n=1}^{\infty} \frac{1}{3^{n}} \sin \left(3^{n+1} x\right)+3 \sum_{n=1}^{\infty} \frac{1}{3^{n}} \sin \left(3^{n} x\right)\right] \\
& =\frac{1}{4}\left[-\sum_{n=1}^{\infty} \frac{1}{3^{n}} \sin \left(3^{n+1} x\right)+3 \sum_{n=0}^{\infty} \frac{1}{3^{n+1}} \sin \left(3^{n+1} x\right)\right]
\end{aligned}
$$

$=\frac{\sin (3 x)}{4}$

and

$$
\begin{aligned}
& V\left(-\frac{1}{3}, 3,3 ; x ; \infty\right)=\frac{1}{2^{3}} \sum_{q=0}^{3}\left(\begin{array}{l}
3 \\
q
\end{array}\right) \sum_{n=1}^{\infty} \frac{(-1)^{n}}{3^{n}} \cos \left[(2 q-3) 3^{n} x\right] \\
& =\frac{1}{4}\left[\sum_{n=1}^{\infty} \frac{(-1)^{n}}{3^{n}} \cos \left(3^{n+1} x\right)+3 \sum_{n=1}^{\infty} \frac{(-1)^{n}}{3^{n}} \cos \left(3^{n} x\right)\right] \\
& =\frac{1}{4}\left[\sum_{n=1}^{\infty} \frac{(-1)^{n}}{3^{n}} \cos \left(3^{n+1} x\right)+3 \sum_{n=0}^{\infty} \frac{(-1)^{n+1}}{3^{n+1}} \cos \left(3^{n+1} x\right)\right] \\
& =-\frac{\cos (3 x)}{4} .
\end{aligned}
$$

The formulas (1.5) and (1.6) are verified once again. By (2.2), it is straightforward that

$$
\begin{aligned}
& U\left(3, \frac{1}{3}, 3 ; x ; \infty\right) \\
& =\sum_{n=1}^{\infty} 3^{n} \frac{(-1)^{3}}{2^{3}} \sum_{q=0}^{3}(-1)^{q+1}\left(\begin{array}{l}
3 \\
q
\end{array}\right) \sin \left[(2 q-3) \frac{1}{3^{n}} x\right] \\
& =\frac{1}{8} \sum_{n=1}^{\infty} 3^{n}\left(-2 \sin \frac{x}{3^{n-1}}+6 \sin \frac{x}{3^{n}}\right) \\
& =\frac{1}{4} \sum_{n=1}^{\infty}\left(3^{n+1} \sin \frac{x}{3^{n}}-3^{n} \sin \frac{x}{3^{n-1}}\right) \\
& =\frac{1}{4}\left[\lim _{n \rightarrow \infty}\left(3^{n+1} \sin \frac{x}{3^{n}}\right)-3 \sin x\right] \\
& =\frac{3}{4}(x-\sin x)
\end{aligned}
$$

and that

$$
\begin{aligned}
& U\left(4, \frac{1}{2}, 4 ; x ; \infty\right)=\sum_{n=1}^{\infty} 4^{n} \frac{1}{2^{4}} \sum_{q=0}^{4}(-1)^{q}\left(\begin{array}{l}
\ell \\
q
\end{array}\right) \cos \left[(2 q-4) \frac{1}{2^{n}} x\right] \\
& =\frac{1}{16} \sum_{n=1}^{\infty} 4^{n}\left[2 \cos \frac{x}{2^{n-2}}-8 \cos \frac{x}{2^{n-1}}+\left(\begin{array}{l}
4 \\
2
\end{array}\right)\right] \\
& =\frac{1}{8} \sum_{n=1}^{\infty} 4^{n}\left(\cos \frac{x}{2^{n-2}}-4 \cos \frac{x}{2^{n-1}}+3\right) \\
& =\frac{1}{8} \sum_{n=1}^{\infty} 4^{n}\left[4\left(1-\cos \frac{x}{2^{n-1}}\right)-\left(1-\cos \frac{x}{2^{n-2}}\right)\right] \\
& =\frac{1}{8} \sum_{n=1}^{\infty}\left[4^{n+1}\left(1-\cos \frac{x}{2^{n-1}}\right)-4^{n}\left(1-\cos \frac{x}{2^{n-2}}\right)\right] \\
& =\frac{1}{8}\left[\lim _{n \rightarrow \infty}\left(4^{n+1}\left(1-\cos \frac{x}{2^{n-1}}\right)\right)-4(1-\cos (2 x))\right] \\
& =\frac{1}{8}\left[8 x^{2}-4(1-\cos (2 x))\right] \\
& =x^{2}-\sin ^{2} x .
\end{aligned}
$$

The formulas (1.4) and (1.1) are recovered alternatively. The proofs of (1.1) to (1.6) are complete.

\section{Remarks}

Remark 3.1. By (2.3) and (2.4), it is not difficult to see that, in order to find summation formulas for $U(\alpha, \beta, \gamma ; x ; \infty)$ and $V(\alpha, \beta, \gamma ; x ; \infty)$, it is sufficient to find summation formulas for the trigonometric series

$u(\alpha, \beta, \gamma, q ; x ; \infty)=\sum_{n=1}^{\infty} \alpha^{n} \sin \left[(2 q-\gamma) \beta^{n} x\right]$ 
and

$v(\alpha, \beta, \gamma, q ; x ; \infty)=\sum_{n=1}^{\infty} \alpha^{n} \cos \left[(2 q-\gamma) \beta^{n} x\right]$

for given $\gamma \in \mathbb{N}$ and for all $0 \leq q \leq \gamma$. Since

$u(\alpha, \beta, \gamma, q ; x ; \infty)=U(\alpha, \beta ;(2 q-\gamma) x)$

and

$v(\alpha, \beta, \gamma, q ; x ; \infty)=V(\alpha, \beta ;(2 q-\gamma) x)$,

it is sufficient to find summation formulas for the infinite trigonometric series $U(\alpha, \beta ; x)$ and $V(\alpha, \beta ; x)$.

Remark 3.2. In [3, Theorem 3.1], Guo and Qi obtained

$\sin (k x)=\sum_{\ell=0}^{k}\left(\begin{array}{l}k \\ \ell\end{array}\right) \sin \frac{\ell \pi}{2} \sin ^{\ell} x \cos ^{k-\ell} x$

and

$\cos (k x)=\sum_{\ell=0}^{k}\left(\begin{array}{l}k \\ \ell\end{array}\right) \cos \frac{\ell \pi}{2} \sin ^{\ell} x \cos ^{k-\ell} x$

for $k \geq 2$. These two identities and the identities (2.1) and (2.2) can be regarded as inversions each other.

\section{References}

[1] W.-C. Chu, Trigonometric formulae via telescoping method, Online J. Anal. Comb. 11 (2016), 8 pages.

[2] I. S. Gradshteyn and I. M. Ryzhik, Table of Integrals, Series, and Products, Translated from the Russian, Translation edited and with a preface by Daniel Zwillinger and Victor Moll, Eighth edition, Revised from the seventh edition, Elsevier/Academic Press, Amsterdam, 2015; Available online at http://dx.doi.org/10.1016/ terdam, 2015; Available online at

[3] B.-N. Guo and F. Qi, On the Wallis formula, Internat. J. Anal. Appl. 8 (2015), no. $1,30-38$ 